\title{
Dynamical $\ell$-boson stars: Generic stability and evidence for nonspherical solutions
}

\author{
Víctor Jaramillo®, ${ }^{1}$ Nicolas Sanchis-Gual $\odot,{ }^{2}$ Juan Barranco $\odot,{ }^{3}$ Argelia Bernal $\odot,{ }^{3}$ Juan Carlos Degollado๑, ${ }^{4}$ \\ Carlos Herdeiro $\oplus^{5}$ and Darío Núñez $\oplus^{1}$ \\ ${ }^{1}$ Instituto de Ciencias Nucleares, Universidad Nacional Autónoma de México, \\ Circuito Exterior C.U., A.P. 70-543, México D.F. 04510, México \\ ${ }^{2}$ Centro de Astrofísica e Gravitação-CENTRA, Departamento de Física, Instituto Superior Técnico-IST, \\ Universidade de Lisboa-UL, Avenida Rovisco Pais 1, 1049-001, Portugal \\ ${ }^{3}$ Departamento de Física, División de Ciencias e Ingenierías, Campus León, Universidad de Guanajuato, \\ León 37150, México \\ ${ }^{4}$ Instituto de Ciencias Físicas, Universidad Nacional Autónoma de México, \\ Apdo. Postal 48-3, 62251, Cuernavaca, Morelos, México \\ ${ }^{5}$ Departamento de Matemática da Universidade de Aveiro and Centre for Research and \\ Development in Mathematics and Applications (CIDMA), \\ Campus de Santiago, 3810-183 Aveiro, Portugal
}

(Received 24 April 2020; accepted 19 May 2020; published 11 June 2020)

\begin{abstract}
$\ell$-boson stars are static, spherical, multifield self-gravitating solitons. They are asymptotically flat, finite energy solutions of Einstein's gravity minimally coupled to an odd number of massive, complex scalar fields. A previous study assessed the stability of $\ell$-boson stars under spherical perturbations, finding that there are both stable and unstable branches of solutions, as for single-field boson stars $(\ell=0)$. In this work we probe the stability of $\ell$-boson stars against nonspherical perturbations by performing numerical evolutions of the Einstein-Klein-Gordon system, with a 3D code. For the timescales explored, the $\ell$-boson stars belonging to the spherical stable branch do not exhibit measurable growing modes. We find, however, evidence of zero modes; that is, nonspherical perturbations that neither grow nor decay. This suggests the branching off toward a larger family of equilibrium solutions: we conjecture that $\ell$-boson stars are the enhanced isometry point of a larger family of static (and possibly stationary), nonspherical multifield selfgravitating solitons.
\end{abstract}

DOI: $10.1103 /$ PhysRevD.101.124020

\section{INTRODUCTION}

Boson stars [1,2] (see [3,4] for reviews) are remarkable gravitational solitons. These self-gravitating, localized energy lumps of a complex, massive scalar field have appealing theoretical properties. A key one is their dynamical stability. For spherical boson stars there is a stable branch of solutions against perturbations. Indeed, a variety of studies including linear perturbation theory [5-7], catastrophe theory [8] and numerical simulations [9-13] agree that boson stars are perturbatively stable, as long as the amplitude of the scalar field is smaller than a critical value. When the latter is attained, boson stars acquire their maximum mass.

Being dynamically stable legitimates inquiring about the possible (astro)physical role of boson stars. Albeit exotic, lacking undisputed observational evidence, boson stars have found important applications in strong gravity and astrophysics. For instance, boson stars provide a common model for a black hole mimicker [14-17]. Being dynamically tractable, one can then compare dynamical spacetime properties, such as waveforms of binary boson star systems, with those of black holes [18-21]. This is particularly timely in view of the recently initiated gravitational-wave astronomy era [22,23], which provides data for both models to be compared with.

A second important application is in relation to a central mystery of contemporary science: the nature of dark matter. An increasing attention has been dedicated to models that consider dark matter as an ultralight bosonic particle [24-27]. The bosonic nature allows this sort of dark matter to form coherent macroscopic excitations. In this context, bosons stars can model, in particular, the core of dark matter galactic halos [28-31].

In their original guise, the Einstein-Klein-Gordon (EKG) model contains a massive, free scalar field, and the solitonic solutions are called mini-boson stars. A variety of generalizations ensued. Boson stars for scalar field theories with self-interactions have been reported, starting with the case of quartic self-interactions considered by Colpi et al. [32]. Spacetime angular momentum was introduced for miniboson stars in $[33,34]$, giving rise to stationary (but not 
static) self-gravitating solitons. A cousin model with a complex, massive vector (rather than scalar) field yields Proca stars [35]. These and other examples use single (complex) field models; however, multifield boson stars have also been reported. One example is given by multistate boson stars [29,30,36]. More recently, multifield boson stars with an arbitrary odd number, $N=2 \ell+1$, $\ell \in \mathbb{N}_{0}$, of equal mass, uncoupled (except through gravity) complex scalar fields with harmonic time dependence were introduced [37]; they are dubbed $\ell$-boson stars and they will be the focus of this paper.

$\ell$-boson stars are described by spherically symmetric and static metrics. For $\ell=0$ they are simply the usual mini-boson stars. For $\ell \geq 1$, the $2 \ell+1$ scalar fields have an angular dependence given by the corresponding $2 \ell+1$ spherical harmonics $Y^{\ell m}$. Then, if the radial dependence for all fields is the same, corresponding to choosing the amplitude of the spherical harmonics equal at all radial distances, static, spherical configurations are obtained, regardless of the energy-momentum tensor of each individual field being angular dependent. This is an example of symmetry noninheritance: the (spherical) spacetime and the (nonspherical) individual matter fields do not share spherical symmetry. The usual boson stars already have a version of symmetry noninheritance: the (time oscillating) scalar field and the (static) spacetime do not share time-translation symmetry. Consistency requires only that the spacetime geometry and the total energy-momentum tensor share the same symmetries, not the individual matter fields.

Generic $\ell$-boson stars have been shown to exhibit similar properties to those of the standard $\ell=0$ stars. In particular, $\ell$-boson stars have a stable branch of solutions against spherical perturbations [38]. The main goal of this paper is to assess the stability of $\ell$-boson stars (in this branch) against generic, nonspherical perturbations. As we shall see, our analysis will show that, in this respect, generic $\ell$-boson star do not exactly mimic the $\ell=0$ case. Although no instabilities are observed, the analysis provides a glimpse of a larger landscape of solutions, of which $\ell$-boson stars are just the enhanced symmetry point.

Departure from spherical symmetry is physically relevant. First, spherical objects-such as $\ell$-boson starsneed to be stable against nonspherical perturbations, in order to be dynamically viable. Second, astrophysical bodies are not, typically, perfectly spherical, in particular due to angular momentum. So one must assess if some perturbations actually deform $\ell$-boson stars into acquiring new degrees of freedom. In this respect, it was recently proposed that multifield boson stars, in the nonrelativistic regime, could have nonspherical stable configurations [39]. This provides an extra motivation to inquire about the behavior of relativistic $\ell$-boson stars under nonspherical perturbations Finally, assessing nonspherical configurations and perturbations often yields a richer phenomenology. As a fruitful example, it was recently found that spinning, single-field miniboson stars are unstable against nonaxisymmetric perturbations, either decaying into a nonrotating boson star or collapsing into a Kerr black hole $[20,40]$. By contrast, spinning Proca stars do not present instabilities under nonaxisymmetric perturbations and furthermore, they can form dynamically [40]. This example shows how the study on nonspherical perturbations unveiled a new relevant dynamical property of boson stars.

We shall investigate the behavior of $\ell$-boson stars under nonspherical perturbations using fully nonlinear numerical simulations of the corresponding Einstein-Klein-Gordon system. As initial data, we use configurations found in [37] which are then perturbed in two different ways. The first type of perturbation tests the stability against nonaxially symmetric perturbations, targeting potential bar-mode instabilities. The second type of perturbation tests the stability against a relative change in the amplitude of the internal fields. In none of the two cases measurable growing modes were found, either by perturbing the total mass density or by perturbing each of the constituent fields, as long the $\ell$-boson star belongs to the stable branch against spherical perturbations. By following the evolution of distortion parameters (defined below) we found, however, evidence for long-lived perturbations, which we interpret as zero modes. These modes, in turn, are interpreted as evidence for a larger family of equilibrium solutions.

Consider the Schwarzschild black hole of vacuum general relativity. It has been shown to be mode stable in the renowned works of Regge and Wheeler [41] and Zerilli [42]. No gravitational perturbations grow. However, a perturbation that carries angular momentum yield not decay. The Schwarzschild solution migrates to a small angular momentum Kerr solution and oscillates around this new ground state. Similarly, a perturbation which electric charge will not decay and the spacetime will oscillate around a small charge Reissner-Nordström solution. These special perturbations are zero modes. Such modes are often found when a spacetime is unstable against some sort of perturbations, at the threshold between stable and unstable modes. An example occurs for the superradiant instability of the Kerr spacetime due to a massive bosonic field. The zero modes indicate the bifurcation of the Kerr family toward a new family of black holes with bosonic hair $[43,44]$. But zero modes can also occur even if there is no instability, as in the Schwarzschild example, indicating, nonetheless, an enlarged family of solutions (Kerr or Reissner-Nordström), of which the initial spacetime (Schwarzschild) is a special case. Thus, one of the outcomes of our analysis is the conjecture that $\ell$-boson stars are the enhanced isometry point of a larger family of static (and possibly stationary), nonspherical multifield selfgravitating solitons.

In the rest of this work we will focus on configurations with $\ell=1$. Such $\ell$-boson stars are described by $N=3$ fields, with $m=-1,0,1$ respectively. In order to follow the 
dynamics of the perturbed system a numerical code that solves the Einstein- $N$-Klein-Gordon system is required. We have used the EINSTEIN TOOLKIT framework [45-47] with the CARPET package $[48,49]$ for mesh-refinement capabilities to achieve our goal.

As a technical step we perform a Cauchy $(3+1)$ decomposition on each scalar field that constitutes the star and solve the full Einstein- $N$-Klein-Gordon system. This is done implementing an arrangement in the EINSTEIN TOOLKIT, a thorn, to solve $N$ scalar fields using finite differences [40].

This paper is organized as follows: Section II addresses the construction of initial data to set up perturbed $\ell$-boson stars. Section III describes the diagnostic tools used to monitor the evolution and some aspects used to decide on whether instabilities are present. The numerical results are described in Sec. IV and in Sec. V our conclusions and final remarks are presented. In this work we use units where $G=1=c$.

\section{INITIAL DATA}

Following previous works on $\ell$-boson stars

[37,38], we consider a set of $N=2 \ell+1$ complex scalar fields, with mass $\mu$ and no self-interaction within the Einstein theory of gravity, for which the energy-momentum tensor is given by:

$$
T_{\alpha \beta}=\sum_{i=1}^{N} T_{\alpha \beta}^{(i)},
$$

where the index $i$ labels each field and the stress-energymomentum for each field is given by

$$
\begin{aligned}
T_{\alpha \beta}^{(i)}= & \left(\nabla_{\alpha} \Phi_{i} \nabla_{\beta} \Phi_{i}^{*}+\nabla_{\beta} \Phi_{i} \nabla_{\alpha} \Phi_{i}^{*}\right) \\
& +g_{\alpha \beta}\left(\nabla_{\sigma} \Phi_{i} \nabla^{\sigma} \Phi_{i}^{*}+\frac{1}{2} \mu^{2}\left|\Phi_{i}\right|^{2}\right) .
\end{aligned}
$$

Complex conjugation is denoted by "**". Following $[37,50]$ we propose a set of scalar fields of the form

$$
\Phi^{(i)}(t, r, \vartheta, \varphi)=\psi_{\ell}(r, t) Y^{\ell m}(\vartheta, \varphi),
$$

where the angular momentum number $\ell$ is fixed, and $m$, which plays the role of index $i$ in Eq. (2.3), takes the values $m=-\ell,-\ell+1, \ldots, \ell$ (hence the total number of fields needed for a fixed value of $\ell$ will be $2 \ell+1$ ), $Y^{\ell m}$ are the spherical harmonics defined over the unitary $2 \mathrm{D}$-sphere. Then we assume that the amplitudes $\psi_{\ell}(r, t)$ are the same for all $m$. It was shown in [37] that if the $N$ fields have all the same amplitude $\psi_{\ell}$, the stress-energy tensor (2.1) has spherical symmetry regardless if the fields have angular dependence. See also [50] and for a detailed discussion on the procedure, see [51].
Assuming the harmonic time dependence

$$
\psi_{\ell}(r, t)=\phi_{\ell}(r) e^{-i \omega t},
$$

where $\phi_{\ell}(r)$ and the frequency $\omega$ are both real-valued, the stress-energy tensor becomes time independent. Under these assumptions it is possible to find self-gravitating static, spherically symmetric equilibrium configurations by solving the EKG system of equations. Those configurations are parametrized by the angular momentum number $\ell$, hence the name, $\ell$-boson stars.

In order to obtain initial data suitable for numerical evolution, we construct equilibrium $\ell$-boson stars, to be subsequently perturbed. Considering a spherically symmetric spacetime with a line element given by:

$d s^{2}=-\alpha(r)^{2} d t^{2}+A(r) d r^{2}+r^{2}\left(d \vartheta^{2}+\sin ^{2} \vartheta d \varphi^{2}\right)$,

where $\alpha$ and $A$, are functions of $r$, and the assumptions mentioned above for $\psi_{\ell}$, the EKG system yields

$$
\begin{aligned}
\partial_{r}^{2} \phi_{\ell}= & -\partial_{r} \phi_{\ell}\left(\frac{2}{r}+\frac{\partial_{r} \alpha}{\alpha}-\frac{\partial_{r} A}{2 A}\right) \\
& +A \phi_{\ell}\left(\mu^{2}+\frac{\ell(\ell+1)}{r^{2}}-\frac{\omega^{2}}{\alpha^{2}}\right), \\
\partial_{r} A=A & \left\{\frac{(1-A)}{r}+4 \pi r A\left[\frac{\left(\partial_{r} \phi_{\ell}\right)^{2}}{A}\right.\right. \\
& \left.\left.+\phi_{\ell}^{2}\left(\mu^{2}+\frac{\ell(\ell+1)}{r^{2}}+\frac{\omega^{2}}{\alpha^{2}}\right)\right]\right\}, \\
\partial_{r} \alpha= & \alpha\left[\frac{(A-1)}{r}+\frac{\partial_{r} A}{2 A}\right. \\
& \left.-4 \pi r A \phi_{\ell}^{2}\left(\mu^{2}+\frac{\ell(\ell+1)}{r^{2}}\right)\right] .
\end{aligned}
$$

By studying the Klein-Gordon equation in the vicinity of $r=0$ one finds that the scalar field behaves as $\phi \sim \phi_{0} r^{\ell}$ in that region. For a fixed value of the angular momentum number $\ell$, a given value of the parameter $\phi_{0}$, and the boundary condition at infinity requesting that $\phi_{\ell}$ decays exponentially, the system of equations (2.6)-(2.8) becomes a nonlinear eigenvalue problem for the frequency $\omega$. We solve this set of equations in a finite size grid by means of a shooting method using the frequency $\omega$ as the shooting parameter. For numerical purposes we take the mass parameter $\mu=1$.

Fig. 1 shows a plot of the Arnowitt-Deser-Misner (ADM) mass $M$ versus the frequency $\omega$ for the $\ell$-boson stars. In Ref. [37] it was shown that $\ell$-boson stars with $\ell>0$ have similar properties to those of single-field miniboson stars, i.e., the $\ell=0$ case. For instance, given a value of $\ell$, the mass $M$ of the equilibrium configurations as a 


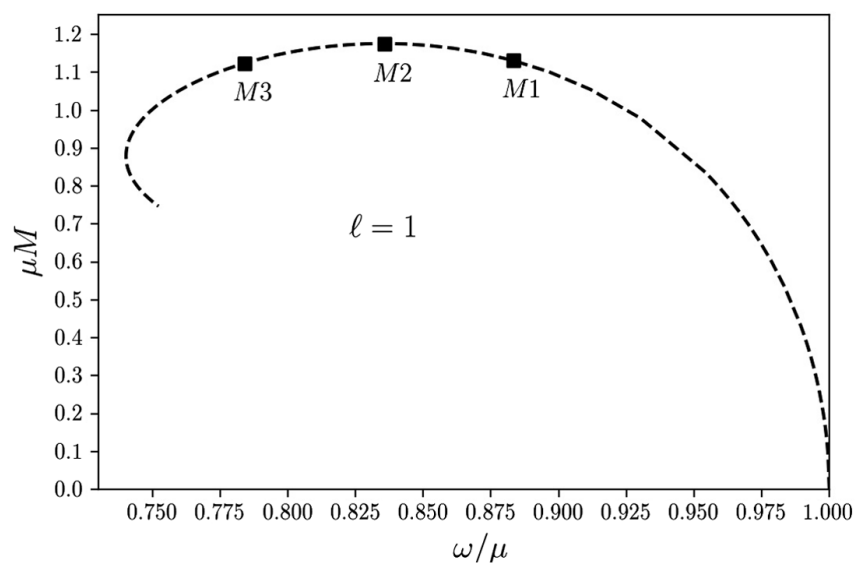

FIG. 1. ADM mass vs frequency for static $\ell$-boson stars. The properties of models $M 1, M 2$ and $M 3$ are listed in Table I.

function of $\omega$ has a maximum, which gets larger as $\ell$ increases, yielding more compact stars. Furthermore, as in the case of 0-boson stars, the maximum value of the mass separates the space of solutions into two branches. These branches correspond to stable and unstable configurations against spherical perturbations, as shown in [38].

As mentioned above, the hypothesis that all the fields must have the same amplitude is essential to keep the spherical symmetry of the configuration. If one wants to consider different amplitudes of each constituent field, the assumption of spherical symmetry has to be relaxed. However, hitherto there has been no evidence that the resulting states may be equilibrium solutions of the Einstein- $N$-Klein-Gordon system. In this work we will show that deviations from spherical symmetry may indeed lead to new equilibrium solutions.

To proceed further with our nonspherical analysis we transform the solutions of the previous system of equations to Cartesian coordinates, $x^{\mu}=(t, r, \vartheta, \varphi) \rightarrow x^{\mu}=$ $(t, x, y, z)$. Then we perform a full nonlinear numerical evolution of the perturbed stationary solutions.

\section{DIAGNOSTICS}

In order to test the stability of the static solutions we perform two different types of perturbations:

(i) The first type consists in perturbing the energy density of the star given by $\rho=n^{\alpha} n^{\beta} T_{\alpha \beta}$, where $n^{\alpha}$ is the four velocity of Eulerian observers in the $3+1$ space time decomposition. The perturbed energy density is obtained by adding a nonspherically symmetric small amplitude term to the homogeneous density in the following way [52]:

$$
\rho=\rho_{0}\left[1+\kappa\left(\frac{x^{2}-y^{2}}{R_{99}^{2}}\right)\right]
$$

where $\rho_{0}$ is the energy density of the equilibrium configuration, obtained from the solution of
Eqs. (2.6)-(2.8), and $R_{99}$ is the radius enclosing $99 \%$ of the configuration's mass. In our simulations we choose $\kappa=0.1$. This type of perturbations could trigger a potential bar-mode instability because it only affects the $I_{x x}$ and $I_{y y}$ components of the quadrupole moment defined as

$$
I_{x x}=\int \rho\left(y^{2}+z^{2}\right) d V, \quad I_{y y}=\int \rho\left(x^{2}+z^{2}\right) d V .
$$

Since the value of $\kappa$ is small $\kappa \ll 1$, this perturbation can be considered linear, initially; more importantly, it breaks the spherical symmetry of the original solution.

(ii) The second type of perturbations consist in varying separately the amplitude of each field. With these perturbations it is possible to study the stability of the stars against variations on each mode $m$ and break the spherical symmetry. We choose the following form

$$
\phi_{\ell, m}=(1+\epsilon) \phi_{\ell}
$$

where $\phi_{\ell}$ is the unperturbed solution of the system of Eqs. (2.6)-(2.8). This perturbation introduces an additional constraint violation, besides the well known numerical error, but its magnitude is controlled by choosing a small $\epsilon$, which, in general, depends on the $\ell, m$-mode. Note that if $\epsilon$ is the same for all $m$, the perturbation is spherical.

In order to assess the stability properties of the stars during the numerical simulation, we monitor the mass of the star, its angular momentum, and its density. We also follow the change in the quadrupole moment of the star, as shown below. Following the technique described in $[52,53]$ to examine the stability of rotating neutron stars, we monitor the behavior of the distortion parameter defined as

$$
\eta_{z}:=\frac{I_{x x}-I_{y y}}{I_{x x}+I_{y y}}
$$

which is a good measure of the magnitude of the bar-mode instability for perturbation (i). This parameter has been used to study the stability of rapidly, differentially rotating stars [52]. It has been observed that when the star is dynamically unstable, $\eta_{z}$ grows exponentially up to a maximum value; then the maximum value of $\eta_{z}$ remains constant on dynamical timescales. For stable stars, on the other hand, the maximum initial value of $\eta_{z}$ remains constant throughout the evolution. Thus the monitoring of $\eta_{z}$ provides a good tool to determine the properties of the star against bar-mode perturbations. In this work we also use 


$$
\eta_{y}:=\frac{I_{x x}-I_{z z}}{I_{x x}+I_{z z}}
$$

as a measure of the deformation of the star.

As further diagnostics, the maximum of the density and the lapse function are used to determine whether the configuration disperses or is undergoing a collapse. We have used the thorn AHFINDER [54] to follow the formation of an apparent horizon (AH) during the evolution. We have also computed the Hamiltonian constraint [55] to check the fourth order convergence of the implementation-see the Appendix for details on this procedure.

\section{TIME EVOLUTION AND NUMERICAL RESULTS}

In this section we present the results from dynamical spacetime simulations from the perturbed $\ell$-boson stars. We have compared the evolution of the equilibrium $\ell$-boson stars with the perturbed stars.

We numerically integrate the EKG system using fourthorder spatial discretization within the EINSTEIN TOOLKIT framework. The EINSTEIN TOOLKIT solves the Einstein equations within the ADM $3+1$ framework and evolves the spacetime using the Baumgarte-Shapiro-ShibataNakamura (BSSN) formulation of the Einstein equations [56] through the MCLACHLAN thorn [57,58]. All the evolutions were made using the $1+\log$ time slicing condition for the lapse $\alpha$, and the Gamma-driver condition for the shift $\beta^{i}$ [59].

We use the Method of Lines thorn to solve the equations in time by using a fourth order Runge-Kutta scheme. The equations for the scalar fields are solved using a finite difference scheme of fourth order. We also employ the mesh refinement capabilities provided by the CARPET arrangements. The fixed mesh refinement grid hierarchy used consists of nested cubes with 3 levels of refinement. The finest is set in such a way that it covers the entire star.

We set the spatial resolution on the finest level to $\{d x, d y, d z\}=0.8$ (and the coarsest to $\{d x, d y, d z\}=3.2$ ) in order to fully capture the properties of the star. We follow the formation of an $\mathrm{AH}$ after the collapse of unstable stars.

More details on the resolution, as well as numerical convergence are given in the Appendix.

The three stationary configurations we chose to illustrate the general behavior of the stars are represented with a square over the curve in Fig. 1 denoted as $(M 1, M 2, M 3)$. Some of the properties of these stars are summarized in Table I.

Both spherical and nonspherical perturbations to the stationary solutions are induced by increasing or decreasing the amplitude of the different constituent fields, see Eq. (3.3). In our case of study, $\ell=1$ and thus, for each configuration $M 1, M 2, M 3$ there are three fields $\phi_{\ell, m}$ : $\left\{\phi_{1,-1}, \phi_{1,0}, \phi_{1,1}\right\}$. We use the position of subindex in the models $\left(M 1_{m=-1, m=0, m=1}\right)$ to label the mode (field) that is
TABLE I. Frequency, radius and ADM mass for the configurations analysed.

\begin{tabular}{lccrc}
\hline \hline Model & $\ell$ & $\omega / \mu$ & $\mu R_{99}$ & $\mu M_{\mathrm{ADM}}$ \\
\hline$M 1$ & 1 & 0.882 & 13.45 & 1.133 \\
$M 2$ & 1 & 0.836 & 12.75 & 1.176 (maximum) \\
$M 3$ & 1 & 0.783 & 7.53 & 1.122 \\
\hline \hline
\end{tabular}

being perturbed. We use + or - to ascribe an increase $(\epsilon>0)$ or decrease $(\epsilon<0)$ of the amplitude, we use 0 to represent that no perturbation was introduced in that mode $(\epsilon=0)$. In this way, for instance, $M 1_{+0-}$ means that $M 1$ has been perturbed in the following way: the first scalar field, $\phi_{1,-1}$, has been perturbed with $\epsilon>0$; the second field $\phi_{1,0}$ has not been perturbed ( $\left.\epsilon=0\right)$, and the third field $\phi_{1,1}$ has been perturbed with $\epsilon<0$. In summary, we have perturbed $M 1, M 2, M 3$ in the following ways: perturbing all fields with the same amplitude as a test (spherical perturbation), introducing a nonaxisymmetric bar-mode perturbation, and finally, we perturbed each constituent field using different amplitudes.

\section{A. Spherical perturbation test}

First, we perform numerical evolutions of the models listed in Table I with spherical perturbations. We induce perturbations in each field of a $\ell$-boson star with all the perturbations having the same amplitude. In this way we guarantee that the spherical symmetry is preserved. This type of perturbations is done in order to compare and validate our results with those found using a spherically symmetric 1D code reported in [38]. While perturbing the initial equilibrium configurations adding perturbations (with a positive or negative value for $\epsilon$ ) that preserve the spherical symmetry, we find that the configuration that was reported to be stable in Ref. [38] (model M1) remains stable in the timescale we reach in the 3D simulations, run $M 1_{000}$. The values of the amplitude of the perturbations for these perturbed configurations are reported in Table II as $M 1_{+++}$ and $M 1_{---}$, in which we perturb each field adding or subtracting $|\epsilon|=0.01$ to each mode.

Our results are also consistent with models of $\ell$-boson stars that are unstable in spherical symmetry. According to the results in [38], the configuration $M 3_{000}$ is unstable in the 1D simulations. When we perturb the amplitudes of the fields adding (run $M 3_{+++}$) or subtracting (run $M 3_{---}$) the same amount, the configuration collapses or migrates to the stable branch respectively, as described in Table II. We monitor the behavior of the metric coefficients during the evolution, and, in particular, we use the lapse and the formation of an $\mathrm{AH}$ as an indicator of the collapse of the star and the formation of a black hole.

The model $M 2$ deserves special mention since it corresponds to the critical solution: the star with maximum mass. We found that perturbations increasing the amplitude of the 
TABLE II. List of simulations performed for the case where all fields are perturbed with the same amplitude (spherical perturbations). These cases are similar to the simulations performed in [38].

\begin{tabular}{lcccc}
\hline \hline Run & $m=+1$ & $m=0$ & $m=-1$ & \\
\hline Model $M 1(\omega / \mu=0.882)$ & & $\epsilon$ & & Collapse \\
$M 1_{000}$ & 0 & 0 & 0 & No \\
$M 1_{+++}$ & +0.01 & +0.01 & +0.01 & No \\
$M 1_{---}$ & -0.01 & -0.01 & -0.01 & No \\
Model $M 2(\omega / \mu=0.836)$ & & $\epsilon$ & & Collapse \\
$M 2_{000}$ & 0 & 0 & 0 & No \\
$M 2_{+++}$ & +0.01 & +0.01 & +0.01 & Yes \\
$M 2_{---}$ & -0.01 & -0.01 & -0.01 & No \\
Model $M 3(\omega / \mu=0.783)$ & & $\epsilon$ & & Collapse \\
$M 3_{000}$ & 0 & 0 & 0 & Yes \\
$M 3_{+++}$ & +0.01 & +0.01 & +0.01 & Yes \\
$M 3_{---}$ & -0.01 & -0.01 & -0.01 & No \\
\hline \hline
\end{tabular}

field ( $\epsilon>0$, run $M 2_{+++}$) make the star collapse whereas perturbations that decrease the amplitude $(\epsilon<0$, run $M 2_{---}$) drive the configuration to a new stable state as described in Table II. These results are consistent with the results reported in [38] for perturbations that increase of decrease the mass of the star.

\section{B. Nonspherical perturbation: Perturbing the energy density}

In order to determine whether $\ell$-boson stars develop a bar mode instability, we took as initial data a stationary model and modified the energy density in accordance with Eq. (3.1). We have performed this analysis for configurations with $\ell=0$ and $\ell=1$, both stable against spherical perturbations. In the case $\ell=0$ we have taken the equilibrium configuration corresponding to $\omega / \mu=0.937$ and for $\ell=1$ the configuration with $\omega / \mu=0.882, M 1$ in Table. I. By choosing $\kappa=0.01$, the momenta of inertia $I_{x x}$ and $I_{y y}$ change by less than $0.5 \%$ with respect to the equilibrium solution, hence we consider that the induced initial perturbation is small. Then we evolve the perturbed system via the Einstein- $N$-Klein-Gordon equations and monitor the behavior of $\eta_{z}$.

In Fig. 2 we show $\eta_{z}$ as a function of time for perturbed and unperturbed configurations for $\ell=0$ (top panel) and $\ell=1$ (bottom panel). For $\ell=0$, the distortion $\eta_{z}$ oscillates around zero for the perturbed case, indicating the star maintains, essentially, the spherical symmetry.

On the other hand, for $\ell=1$ in the case where the perturbation was included, the initial perturbation induces a small deviation from spherical symmetry therefore $\eta_{z}$ acquires a nontrivial value during the evolution. This nonzero value of $\eta_{z}$ indicates that the shape of the star deviates from spherical and becomes oblate.
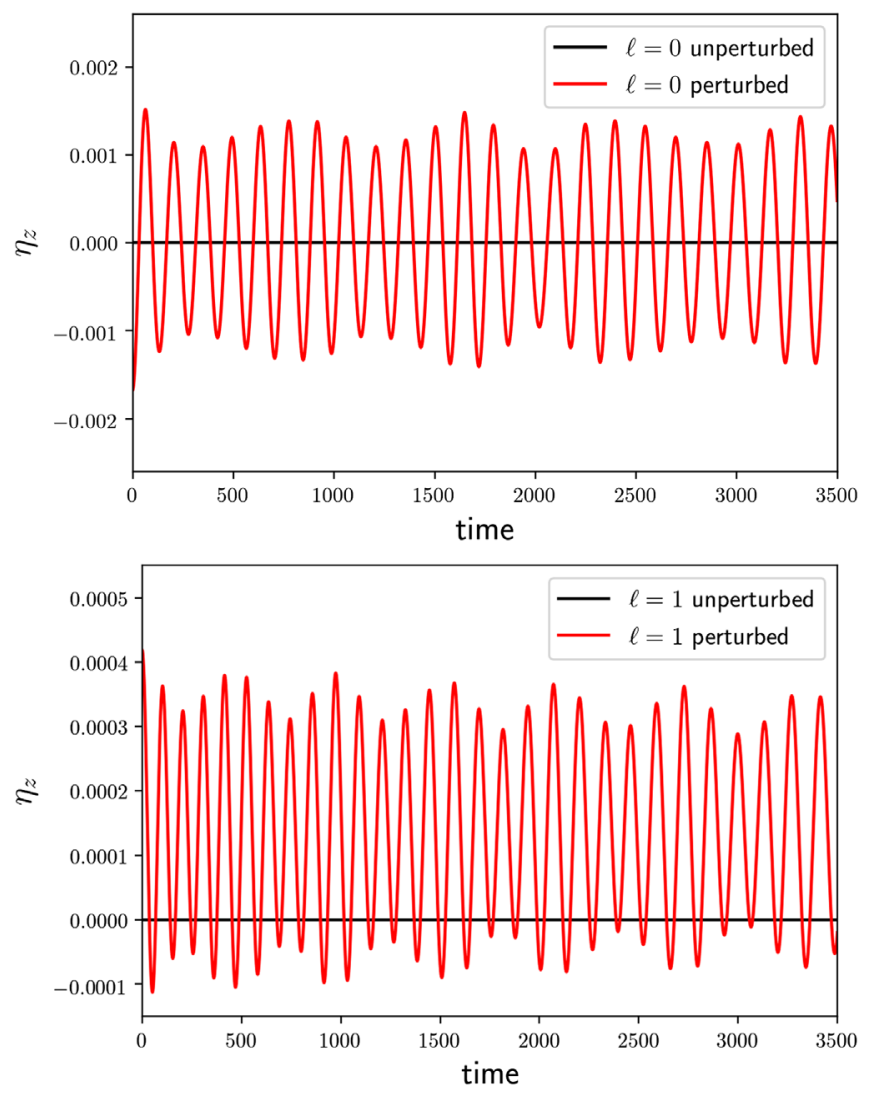

FIG. 2. Evolution of $\eta_{z}$ as a function of time for unperturbed (black solid line) and perturbed density as defined by Eq. (3.1) (red solid line) with $\kappa=0.01$. Top panel: the unperturbed configuration is a single-field boson star $(\ell=0)$. Bottom panel: the unperturbed configuration is a multifield boson $\operatorname{star}(\ell=1)$. In neither perturbed case has a bar instability been observed. Instead, a long lived departure from spherical symmetry occurs for $\ell=1$, but not for $\ell=0$, as long as $\eta_{z} \neq 0$. In contrast, the unperturbed configurations preserve spherical symmetry as $\eta_{z}$ oscillates around zero in both cases.

During the evolution time considered $(t \sim 3500)$ we did not find any signal of a bar-mode instability for the models considered: no exponential growth in $\eta_{z}$ was measured. Most importantly $\eta_{z}$ does not grow as it happens for unstable stars [40]. This change in the shape of the $\ell=1$ configuration, illustrated by $\eta_{z} \neq 0$, is compared with the case where $M 1$ is not perturbed (black solid line). For the unperturbed case, $\eta_{z}$ simply oscillates around zero. The conclusion, therefore, is that the perturbed configuration lingers, neither collapsing nor dissipating, thus showing a nonspherical distribution that is either stable or long-lived, without signs of instability. It is worth emphasizing the key difference with the $\ell=0$ case, for which the evolution oscillates around a spherical distribution, in agreement with the fact that such a distribution is the only equilibrium configuration.

We found that after some time, the stars acquire a small linear momentum due to the numerical error and thus the 
deviation parameters can not be obtained accurately. Once this becomes noticeable we stop the evolution.

\section{Nonspherical perturbation: Perturbing the amplitude of each mode}

In this section, we describe the evolutions we have performed implementing nonspherical perturbation by varying the amplitude of each field of the $\ell$-boson star.

\section{Nonspherical perturbation of M1}

In order to illustrate the procedure to perturb the star, let us consider first model $M 1$. Different perturbations have been applied to $M 1$ and all them are summarized in Table III. Subscripts indicate which fields have been perturbed and if the amplitude is increased by $\epsilon>0$ (subscript + ), decreased by $\epsilon<0$ (subscript - ) or it has been left without perturbation $\epsilon=0$ (subscript 0 ), as described before.

All our evolutions show that $M 1$ remains stable without collapsing (no $\mathrm{AH}$ was found), independently of the perturbation. Thus, from the results summarized in Table III we can conclude that the configurations in the stable branch (against spherical perturbations), that is, to the right of configuration $M 2$ in Fig. 1, are also stable under nonspherical perturbations, against collapse.

Let us now turn to another result that can be extracted by studying the distortion parameters $\eta_{z}$ and $\eta_{y}$. For spherical configurations and for those configurations that are spherically perturbed, these are zero. On the other hand, for nonspherical perturbations a small deviation from spherical symmetry is induced. In other words, nontrivial values of $\eta_{y}$ are obtained throughout the evolution of $M 1$. This behavior of $\eta_{y}$ as a function of time is shown in Fig. 3. Indeed, nonzero values of $\eta_{y}$ for the evolution of $M 1_{+00}, M 1_{00,+}$, $M 1_{-00}, M 1_{00-}, M 1_{0+0}$, and $M 1_{0-0}$, are obtained. No

TABLE III. List of simulations performed for model $M 1$ under the second type of perturbations. The parenthesis indicate a larger amplitude on the perturbations. Notice that gravitational collapse was not observed in any of the simulations.

\begin{tabular}{lcccc}
\hline \hline Model $M 1(\omega / \mu=0.882)$ & \multicolumn{5}{c}{$\epsilon$} & Collapse \\
\hline Run & $m=+1$ & $m=0$ & $m=-1$ & \\
\hline$M 1_{+00}$ & +0.01 & 0 & 0 & No \\
$M 1_{-00}$ & -0.01 & 0 & 0 & No \\
$M 1_{0+0}$ & 0 & +0.01 & 0 & No \\
$M 1_{0-0}$ & 0 & -0.01 & 0 & No \\
$M 1_{00+}$ & 0 & 0 & +0.01 & No \\
$M 1_{00-}$ & 0 & 0 & -0.01 & No \\
$M 1_{++0}$ & +0.01 & +0.01 & 0 & No \\
$M 1_{+-+}$ & +0.01 & -0.01 & +0.01 & No \\
$M 1_{+0+}$ & +0.01 & 0 & +0.01 & No \\
$M 1_{-0+}$ & -0.01 & 0 & +0.01 & No \\
$M 1_{0(-) 0}$ & 0 & -0.1 & 0 & No \\
\hline \hline
\end{tabular}

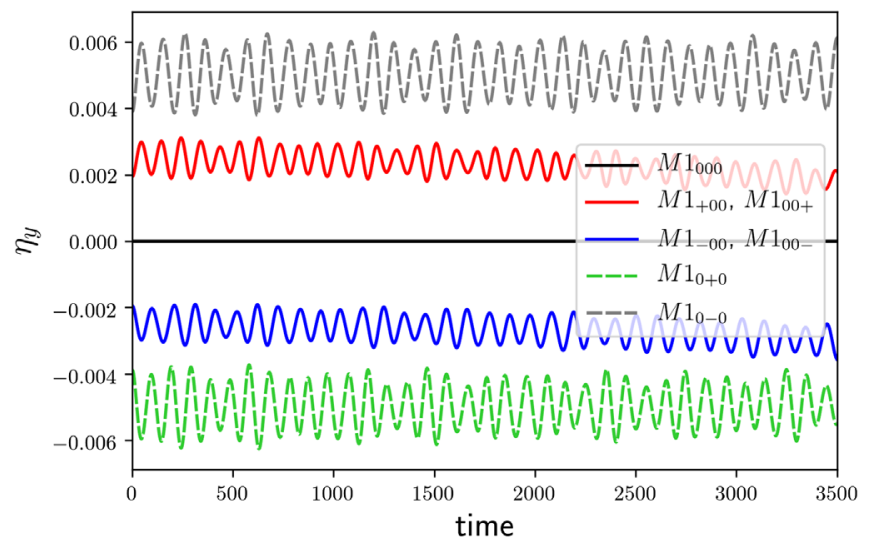

FIG. 3. Distortion parameter $\eta_{y}$, for model $M 1$ for runs shown in Table III. Departure from spherical symmetry is shown for those configurations that have been perturbed differently in all the three fields $\phi_{1,-1}, \phi_{1,0}$ and $\phi_{1,1}$. On the contrary, the configuration $M 1_{000}$ has the same perturbation for all the fields (spherical perturbation), and it shows $\eta_{y}=0$ at all times.

instability is observed, but the deformation does not die off either. These long-lived deformed configurations, arising as dynamical solutions of the Einstein- $N$-Klein-Gordon with three fields with different $m$ are not spherically symmetric. The corresponding perturbations appear to be zero modes, suggesting a larger family of solutions.

As expected, we observe that the equilibrium configuration $M 1_{000}$ has $\eta_{y}=0$ at all times of the evolution. Besides, we have found that the behavior of $\eta_{y}$ and $\eta_{z}$ during the evolution is the same for perturbations in the modes $m=1$ and $m=-1$ with the same values of $\epsilon$. This suggests that the resulting configurations are axially symmetric.

Finally, we report in Fig. 4 the time evolution of the total mass of $M 1$ under different spherical and nonspherical perturbations. As expected, the mass of the perturbed

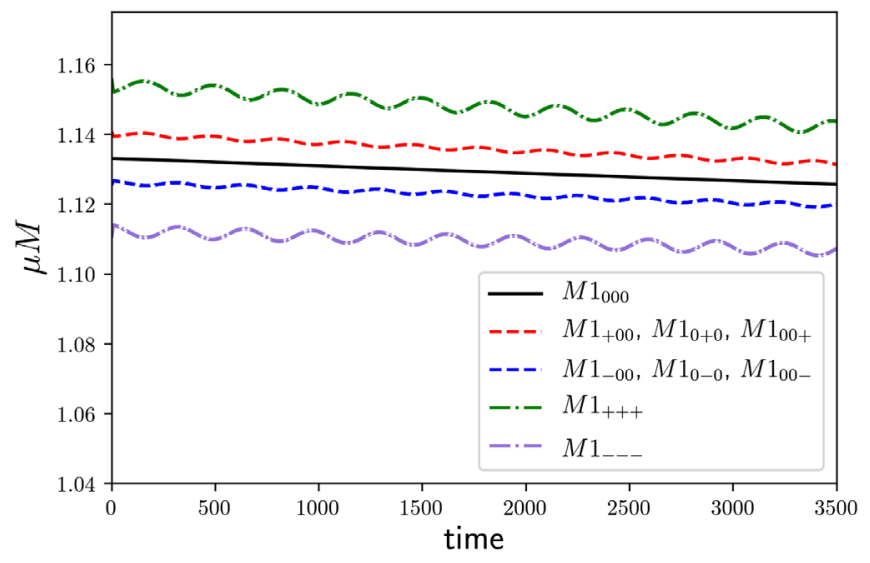

FIG. 4. Evolution of the mass of the model $M 1$ subjected to spherical and nonspherical perturbations listed in Tables II and III respectively. The mass, as expected, is increased for those perturbations with $\epsilon>0$ and decreases when $\epsilon<0$. 
configurations decreases or increases when $\epsilon<0$ or $\epsilon>0$. Notice, however, the change in mass is the same whether we perturb the $m=-1$ or $m=1$ modes, for the same the sign of $\epsilon$. This fact supports the assertion that the resulting configurations are axially symmetric.

The total mass of the models decreases with time, showing a small drift, even for the unperturbed solution. We have checked that this drift is due to the numerical error, since it is reduced when the grid resolution is increased (see Appendix A).

Fig. 5 displays a series of snapshots of projections in the planes $x y$ and $x z$ of the energy density for the run $M 1_{0(-) 0}$ (With a large amplitude in the perturbation in the mode $m=0$ ). The initial perturbation is introduced in the mode $m=0$, decreasing the mass of the star and inducing a small deformation. Notice that we have taken the largest perturbation presented in this section (model $M 1_{0(-) 0}$ ), so that the deformation can be appreciated in these projections of the energy density. At later times the system evolves and settles down into a configuration without collapsing or exploding. Although the configuration looks almost spherical, the value of $\eta_{y}$ at late times is slightly different from zero $\left(\eta_{y} \sim 0.05\right)$. We have evolved this configuration for $t \sim$ 10000 and it remains in the same state not showing any signs of instability, or returning to a $\ell$-boson star.

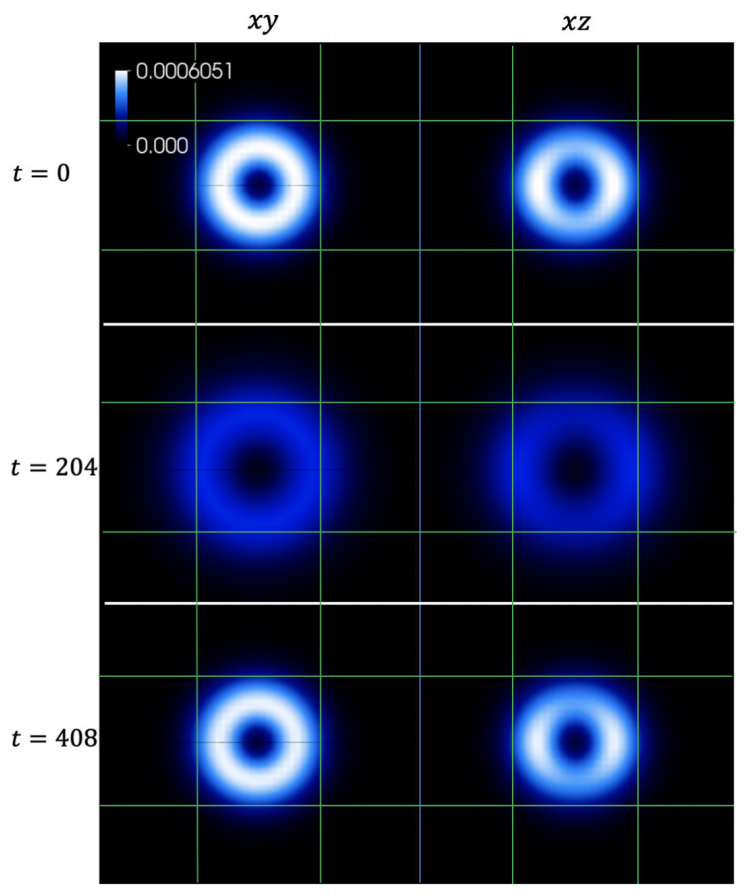

FIG. 5. Three snapshots of the projection of the rest mass density in two planes. In the second snapshot the star expands and thus the maximum value of the density decreases. In the third snapshot the star returns to its original state. This repetitive behavior is present during all the evolution time. The mesh represent a box with sides $\frac{1}{2} R_{99}$ of the unperturbed star.
At this point it is important to mention that perturbations in the mode $m=0$ do not modify the value of the total angular momentum, while perturbations in the modes $m=1$ or $m=-1$ do. Specifically, $M 1_{-00}$ and $M 1_{00+}$ $\left(M 1_{+00}\right.$ and $\left.M 1_{00_{-}}\right)$have a positive (negative), nontrivial and constant value of total angular momentum. As we could expect, runs like $M 1_{+0+}$ have zero angular momentum. This particular result was also obtained for the perturbations of the models that will be presented below.

\section{Nonspherical perturbation of M2 and M3}

The results of the previous section indicate that those configurations $(M 1)$ that are stable under spherical perturbations do not show nonspherical growing modes. Furthermore, perturbations to the fields $\phi_{1,-1}$ and $\phi_{1,1}$ applied to those configurations provide evidence for zero modes, producing new equilibrium configurations that are dynamically stable, and have small departures from the spherical configurations.

Now we are interested in studying nonspherical perturbations on configurations that might undergo gravitational collapse to a black hole. Those configurations are $M 2$ and M3. In particular $M 2$, as we have mentioned, corresponds to the critical configuration with maximum ADM mass. Configuration $M 2$, as is shown in Sec. IV A and reported in the literature [38], divides stable from unstable configurations. The latter are those configurations that can collapse into a black hole. We now go one step further and study them under nonspherical perturbations.

The list of perturbation applied to each field of $M 2$ is summarized in Table IV and the result of the evolution is reported in the fifth column of the same table. The results confirm that $M 2$ is the configuration that separates stable from unstable configurations. Indeed, as it can be observe in the results of Table IV, all those configurations which

TABLE IV. List of simulations performed from the model M2 under nonspherical perturbations. Those configurations that increased the total mass by the addition of the perturbation did collapse to a black hole. Configurations that did not change the total mass or did not decrease the total mass of the configuration did not collapse.

\begin{tabular}{lcccc}
\hline \hline Model $M 2(\omega / \mu=0.836)$ & \multicolumn{3}{c}{$\epsilon$} & Collapse \\
\hline Run & $m=+1$ & $m=0$ & $m=-1$ & \\
\hline$M 2_{+00}$ & +0.01 & 0 & 0 & Yes \\
$M 2_{-00}$ & -0.01 & 0 & 0 & No \\
$M 2_{0+0}$ & 0 & +0.01 & 0 & Yes \\
$M 2_{0-0}$ & 0 & -0.01 & 0 & No \\
$M 2_{00+}$ & 0 & 0 & +0.01 & Yes \\
$M 2_{00-}$ & 0 & 0 & -0.01 & No \\
$M 2_{++0}$ & +0.01 & +0.01 & 0 & Yes \\
$M 2_{+-+}$ & +0.01 & -0.01 & +0.01 & Yes \\
$M 2_{+0+}$ & +0.01 & 0 & +0.01 & Yes \\
$M 2_{-0+}$ & -0.01 & 0 & +0.01 & No \\
\hline \hline
\end{tabular}


TABLE V. List of simulations performed form the model M3 under nonspherical perturbations. Those configurations that increased the total mass by the addition of the perturbation did collapse to a black hole. Configurations that decreased the total mass of the initial configuration, migrated to the stable branch. The run $M 3_{-0+}$ that did not change the total mass did collapse to a black hole.

\begin{tabular}{lcccc}
\hline \hline Model $M 3(\omega / \mu=0.783)$ & \multicolumn{5}{c}{$\epsilon$} & Collapse \\
\hline Run & $m=+1$ & $m=0$ & $m=-1$ & \\
\hline$M 3_{+00}$ & +0.01 & 0 & 0 & Yes \\
$M 3_{-00}$ & -0.01 & 0 & 0 & No \\
$M 3_{0+0}$ & 0 & +0.01 & 0 & Yes \\
$M 3_{0-0}$ & 0 & -0.01 & 0 & No \\
$M 3_{00+}$ & 0 & 0 & +0.01 & Yes \\
$M 3_{00-}$ & 0 & 0 & -0.01 & No \\
$M 3_{++0}$ & +0.01 & +0.01 & 0 & Yes \\
$M 3_{+-+}$ & +0.01 & -0.01 & +0.01 & Yes \\
$M 3_{+0+}$ & +0.01 & 0 & +0.01 & Yes \\
$M 3_{-0+}$ & -0.01 & 0 & +0.01 & Yes \\
\hline \hline
\end{tabular}

have perturbations that increased the total mass of the configuration undergo a collapse, while those configurations which have been perturbed and reduced the total mass of the configuration did not collapse to a black hole. In this respect, run $M 2_{-0+}$ is of special interest. The perturbation did not change the total mass of the configuration, and the result of their evolution is that it did not collapse.

Finally, we have considered nonspherical perturbations of model M3. The results of the evolution of the different models studied are summarized in Table V. This configuration is on the so called unstable branch. The results mimic, to some extent, those observed for $M 2$. Perturbations that reduced the total mass of the configuration led to a migration to the stable branch. On the other hand, those that increased the mass of the configuration let to a collapse into a black hole. But a key difference is seen for $M 3_{-0+}$. This perturbation did not change the total mass of the configuration, and contrary to $M 2_{-0+}$, it did collapse to a black hole. This result, combined with the spherical perturbations mentioned in IVA, further confirms the special status of the maximum mass configuration $M 2$ : it marks the threshold of unstable configurations of $\ell$-boson stars, for both spherical and nonspherical perturbations.

\section{DISCUSSION AND OUTLOOK}

In this paper we performed dynamical simulations in the fully nonlinear EKG model to investigate the stability of $\ell$-boson stars. Unlike previous works we have considered nonspherical perturbations. An expected result is that those configurations known to be unstable under spherical perturbations, are also unstable under more general perturbations. The most interesting question, however, was if the configurations known to be stable under spherical perturbations would remain stable under more general ones. Here, our conclusions are two-fold. First, no growing modes have been measured in our simulations. In this sense $\ell$-boson stars are stable against nonspherical perturbations. However, when deformed away from sphericity, $\ell$-boson stars do not return to a spherical state. They appear to oscillate around a new (slightly) nonspherical state. We take this as evidence that new, multifield, equilibrium configurations of the Einstein- $N$-Klein Gordon system exist, which are nonspherical. This conjecture is our second conclusion.

If our conjecture is proven correct, the spherically symmetric $\ell$-boson stars are only an enhanced isometry point of a larger family of solutions of the Einstein- $\mathrm{N}$-Klein Gordon. As discussed in the introduction, this is analogous to the Schwarzschild BH being the isometry enhancement point of the Kerr family. It is well known that the Kerr solution brings about qualitatively novel features with respect to the Schwarzschild solution. So it will be quite interesting to understand the novelties brought by the enlarged family of solutions that this work is suggesting.

The conjecture on the existence of these new nonspherical, multifield configurations can be tested by solving the Einstein- $\mathrm{N}$-Klein Gordon system for static or stationary (i.e., spinning) configurations, without assuming spherical symmetry. Research in this direction is already ongoing.

\section{ACKNOWLEDGMENTS}

We thank Miguel Alcubierre, Alberto Diez, Miguel Meguevand, Eugen Radu and Olivier Sarbach, for stimulating discussions. This work was supported in part by the CONACYT Network Project No. 280908 "Agujeros Negros y Ondas Gravitatorias", by DGAPA-UNAM through Grants No. IN110218, No. IA103616, No. IN105920, by the European Union's Horizon 2020 research and innovation (RISE) program H2020-MSCARISE-2017 Grant No. FunFiCO-777740, by the Center for Research and Development in Mathematics and Applications (CIDMA) through the Portuguese Foundation for Science and Technology (FCTFundacao para a Ciência e a Tecnologia), references UIDB/04106/2020 and UIDP/04106/2020, and by the projects No. PTDC/FIS-OUT/28407/2017, No. CERN/ FIS-PAR/0027/2019 and No. UID/FIS/00099/2020 (CENTRA). The authors would like to acknowledge networking support by the COST Action CA16104. V. J. acknowledge support from CONACYT.

\section{APPENDIX: CODE VALIDATION}

For run $M 1_{000}$ we report the time evolution of the mass and violations of the Hamiltonian constraint, with different resolutions, $\{d x, d y, d z\}=3.2,\{d x, d y, d z\}=\sqrt{2} 1.6$ and $\{d x, d y, d z\}=1.6$, where $d x, d y$ and $d z$ are the sizes of the coarsest level of refinement. 


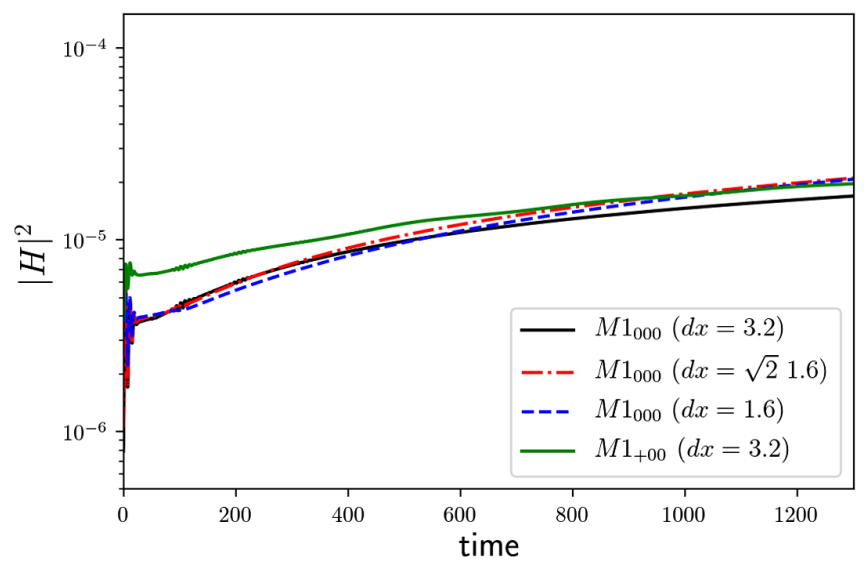

FIG. 6. Convergence for run $M 1_{000}$ : Evolution of the L2-norm of the Hamiltonian constraint for three different resolutions rescaled to show fourth order convergence. Green line shows L2-norm of the Hamiltonian constrain for a perturbed run, initially, the violation of the constraint due to the perturbation is evident, as time passes the magnitude of the error is comparable with the error of the unperturbed runs.

The L2-norm of the Hamiltonian constraint, given by $|H|^{2}=\sqrt{\frac{\sum_{i=1}^{N} H_{i}^{2}}{N}}$, where $N$ is the number of points in the grid, is shown in Fig. 6. Here we conclude that the constraint equations converge as $H$ reduces when the resolution is increased, the black (solid) and the blue (dashed) line have been multiplied by the factors 4 and

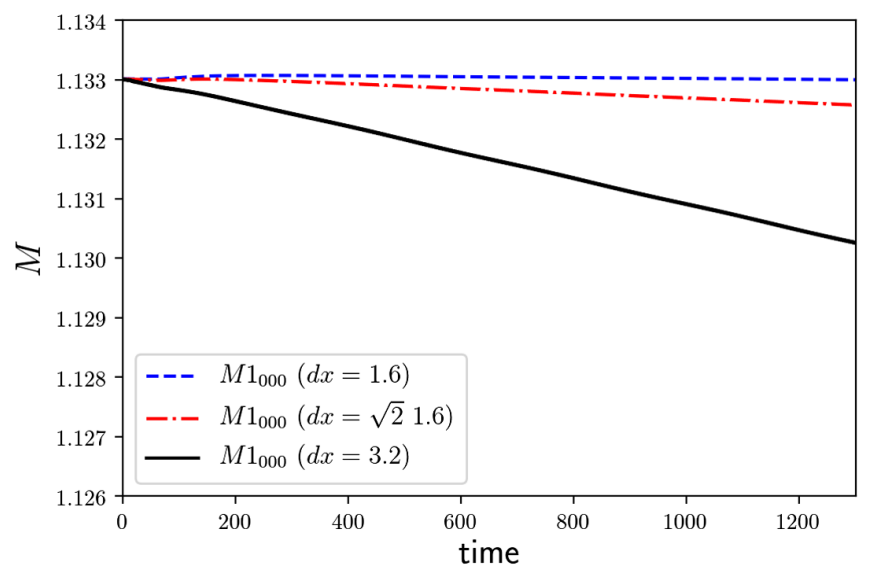

FIG. 7. Convergence for run $M 1_{000}$ : Evolution of the mass for three different resolutions.

16 , showing fourth order convergence. The low resolution (solid line, coarsest grid $d x=3.2$ ) corresponds to the one used in all the simulations presented in this work. The L2norm of $H$ increases with time, however tends to a constant value which approaches zero as $\{d x, d y, d z\} \rightarrow 0$.

We plot the mass for run $M 1_{000}$. As the resolution is increased the mass converge to a constant value and the overall drift is reduced. In Figs. 6 and 7 we have plotted until time equal to 1300 , however the $(\{d x, d y, d z\}=3.2)$ simulation extends up to $t \sim 3500$, where the final total mass differs from the initial value by $0.6 \%$.
[1] D. J. Kaup, Klein-gordon geon, Phys. Rev. 172, 1331 (1968).

[2] R. Ruffini and S. Bonazzola, System of self-gravitating particles in general relativity and the concept of an equation of state, Phys. Rev. 187, 1767 (1969).

[3] F. E. Schunck and E.W. Mielke, General relativistic boson stars, Classical Quantum Gravity 20, R301 (2003).

[4] Steven L. Liebling and Carlos Palenzuela, Dynamical boson stars, Living Rev. Relativity 15, 6 (2012).

[5] T. D. Lee and Y. Pang, Stability of mini-boson stars, Nucl. Phys. B315, 477 (1989).

[6] Marcelo Gleiser, Stability of boson stars, Phys. Rev. D 38, 2376 (1988); Erratum, Phys. Rev. D 39, 1257 (1989).

[7] M. Gleiser and R. Watkins, Gravitational stability of scalar matter, Nucl. Phys. B319, 733 (1989).

[8] Fyodor V. Kusmartsev, Eckehard W. Mielke, and Franz E. Schunck, Stability of neutron and boson stars: A new approach based on catastrophe theory, Phys. Lett. A 157, 465 (1991).

[9] S. H. Hawley and M. W. Choptuik, Boson stars driven to the brink of black hole formation, Phys. Rev. D 62, 104024 (2000).
[10] E. Seidel and W. Suen, Dynamical evolution of boson stars: Perturbing the ground state, Phys. Rev. D 42, 384 (1990).

[11] F.S. Guzman, The three dynamical fates of bosons stars, Rev. Mex. Fis. 55, 321 (2009), http://www .scielo.org.mx/scielo.php?script=sci_abstract\&pid=S0035001X2009000400011\&lng=es\&nrm=iso.

[12] N. Sanchis-Gual, C. Herdeiro, E. Radu, J. C. Degollado, and J. A. Font, Numerical evolutions of spherical proca stars, Phys. Rev. D 95, 104028 (2017).

[13] F. Di Giovanni, N. Sanchis-Gual, C. A. R. Herdeiro, and J. A. Font, Dynamical formation of proca stars and quasistationary solitonic objects, Phys. Rev. D 98, 064044 (2018).

[14] E. W. Mielke and F. E. Schunck, Boson stars: Alternatives to primordial black holes?, Nucl. Phys. B564, 185 (2000).

[15] D. F. Torres, S. Capozziello, and G. Lambiase, A supermassive boson star at the galactic center?, Phys. Rev. D 62, 104012 (2000).

[16] F. S. Guzman and J. M. Rueda-Becerril, Spherical boson stars as black hole mimickers, Phys. Rev. D 80, 084023 (2009).

[17] H. Olivares, Z. Younsi, C. M. Fromm, M. De Laurentis, O. Porth, Y. Mizuno, H. Falcke, M. Kramer, and L. Rezzolla, 
How to tell an accreting boson star from a black hole, arXiv:1809.08682.

[18] C. Palenzuela, I. Olabarrieta, L. Lehner, and S. L. Liebling, Head-on collisions of boson stars, Phys. Rev. D 75, 064005 (2007).

[19] C. Palenzuela, L. Lehner, and S. L. Liebling, Orbital dynamics of binary Boson star systems, Phys. Rev. D 77, 044036 (2008).

[20] M. Bezares, C. Palenzuela, and C. Bona, Final fate of compact boson star mergers, Phys. Rev. D 95, 124005 (2017).

[21] C. Palenzuela, P. Pani, M. Bezares, V. Cardoso, L. Lehner, and S. Liebling, Gravitational wave signatures of highly compact boson star binaries, Phys. Rev. D 96, 104058 (2017).

[22] B. P. Abbott et al., Observation of Gravitational Waves from a Binary Black Hole Merger, Phys. Rev. Lett. 116, 061102 (2016).

[23] B. P. Abbott et al., GW150914: First results from the search for binary black hole coalescence with Advanced LIGO, Phys. Rev. D 93, 122003 (2016).

[24] T. Matos, F. S. Guzman, and L. A. Urena-Lopez, Scalar field as dark matter in the universe, Classical Quantum Gravity 17, 1707 (2000).

[25] T. Matos and L. A. Urena-Lopez, A further analysis of a cosmological model of quintessence and scalar dark matter, Phys. Rev. D 63, 063506 (2001).

[26] W. Hu, R. Barkana, and A. Gruzinov, Fuzzy Cold Dark Matter: The Wave Properties of Ultralight Particles, Phys. Rev. Lett. 85, 1158 (2000).

[27] L. Hui, J. P. Ostriker, S. Tremaine, and E. Witten, Ultralight scalars as cosmological dark matter, Phys. Rev. D 95, 043541 (2017).

[28] J.-W. Lee and I.-G. Koh, Galactic halos as boson stars, Phys. Rev. D 53, 2236 (1996).

[29] A. Bernal, J. Barranco, D. Alic, and C. Palenzuela, Multistate boson stars, Phys. Rev. D 81, 044031 (2010).

[30] L. A. Urena-Lopez and A. Bernal, Bosonic gas as a Galactic dark matter halo, Phys. Rev. D 82, 123535 (2010).

[31] H.-Yu Schive, T. Chiueh, and T. Broadhurst, Cosmic structure as the quantum interference of a coherent dark wave, Nat. Phys. 10, 496 (2014).

[32] M. Colpi, S. L. Shapiro, and I. Wasserman, Boson Stars: Gravitational Equilibria of Self-Interacting Scalar Fields, Phys. Rev. Lett. 57, 2485 (1986).

[33] S. Yoshida and Y. Eriguchi, Rotating boson stars in general relativity, Phys. Rev. D 56, 762 (1997).

[34] F. E. Schunck and E. W. Mielke, Rotating boson star as an effective mass torus in general relativity, Phys. Lett. A 249 , 389 (1998).

[35] R. Brito, V. Cardoso, C. A. R. Herdeiro, and E. Radu, Proca stars: Gravitating bose-einstein condensates of massive spin 1 particles, Phys. Lett. B 752, 291 (2016).

[36] H.-B. Li, S. Sun, T.-T. Hu, Y. Song, and Y.-Q. Wang, Rotating multistate boson stars, Phys. Rev. D 101, 044017 (2020).

[37] M. Alcubierre, J. Barranco, A. Bernal, J. C. Degollado, A. Diez-Tejedor, M. Megevand, D. Nunez, and O. Sarbach, $\ell$-boson stars, Classical Quantum Gravity 35, 19LT01 (2018).
[38] M. Alcubierre, J .Barranco, A. Bernal, J. Carlos Degollado, A. Diez-Tejedor, M. Megevand, D. Núñez, and O. Sarbach, Dynamical evolutions of $\ell$-boson stars in spherical symmetry, Classical Quantum Gravity 36, 215013 (2019).

[39] F. S. Guzmán and L. A. Ureña-López, Gravitational atoms: General framework for the construction of multistate axially symmetric solutions of the Schrödinger-Poisson system, Phys. Rev. D 101, 081302 (2020).

[40] N. Sanchis-Gual, F. Di Giovanni, M. Zilhão, C. Herdeiro, P. Cerdá-Durán, J. A. Font, and E. Radu, Nonlinear Dynamics of Spinning Bosonic Stars: Formation and Stability, Phys. Rev. Lett. 123, 221101 (2019).

[41] T. Regge and J. Wheeler, Stability of a Schwarzschild singularity, Phys. Rev. 108, 1063 (1957).

[42] F. J. Zerilli, Effective Potential for Even Parity ReggeWheeler Gravitational Perturbation Equations, Phys. Rev. Lett. 24, 737 (1970).

[43] C. A. R. Herdeiro and Eugen Radu, Kerr Black Holes with Scalar Hair, Phys. Rev. Lett. 112, 221101 (2014).

[44] C. Herdeiro, E. Radu, and H. Rúnarsson, Kerr Black Holes with Proca Hair, Classical Quantum Gravity 33, 154001 (2016).

[45] Einstein Toolkit: Open software for relativistic astrophysics, http://einsteintoolkit.org/.

[46] F. Löffler, J. Faber, E. Bentivegna, T. Bode, P. Diener, R. Haas, I. Hinder, B. C. Mundim, C. D. Ott, E. Schnetter, G. Allen, M. Campanelli, and P. Laguna, The Einstein Toolkit: A community computational infrastructure for relativistic astrophysics, Classical Quantum Gravity 29, 115001 (2012).

[47] M. Zilhão and F. Löffler, An introduction to the Einstein toolkit, Int. J. Mod. Phys. A 28, 1340014 (2013).

[48] E. Schnetter, S. H. Hawley, and I. Hawke, Evolutions in 3D numerical relativity using fixed mesh refinement, Classical Quantum Gravity 21, 1465 (2004).

[49] http://www.carpetcode.org/, Carpet: Adaptive Mesh Refinement for the Cactus Framework.

[50] I. Olabarrieta, J. F. Ventrella, M. W. Choptuik, and W. G. Unruh, Critical behavior in the gravitational collapse of a scalar field with angular momentum in spherical symmetry, Phys. Rev. D 76, 124014 (2007).

[51] B. Carvente, V. Jaramillo, J. C. Degollado, D. Núñez, and O. Sarbach, Traversable $\ell$-wormholes supported by ghost scalar fields, Classical Quantum Gravity 36, 235005 (2019).

[52] M. Saijo, M. Shibata, T. W. Baumgarte, and S. L. Shapiro, Dynamical bar instability in rotating stars: Effect of general relativity, Astrophys. J. 548, 919 (2001).

[53] M. Shibata and H. Yoshino, Bar-mode instability of rapidly spinning black hole in higher dimensions: Numerical simulation in general relativity, Phys. Rev. D 81, 104035 (2010).

[54] P. Diener, A new general purpose event horizon finder for 3d numerical spacetimes, Classical Quantum Gravity 20, 4901 (2003).

[55] E. Gourgoulhon, 3+1 Formalism in General Relativity, Bases of Numerical Relativity (Springer, Heidelberg, 2012).

[56] T. W. Baumgarte and S.L. Shapiro, On the numerical integration of Einstein's field equations, Phys. Rev. D 59, 024007 (1998). 
[57] D. Brown, P. Diener, O. Sarbach, E. Schnetter, and M. Tiglio, Turduckening black holes: An analytical and computational study, Phys. Rev. D 79, 044023 (2009).

[58] C. Reisswig, C. D. Ott, U. Sperhake, and E. Schnetter, Gravitational wave extraction in simulations of rotating stellar core collapse, Phys. Rev. D 83, 064008 (2011).

[59] M. Alcubierre, Introduction to 3+1 Numerical Relativity (Oxford University Press, New York, 2008). 